\title{
Item exposure control for multidimensional computer adaptive testing under maximum likelihood and expected a posteriori estimation
}

\author{
Alan R. Huebner ${ }^{1} \cdot$ Chun Wang $^{2} \cdot$ Kari Quinlan $^{1} \cdot$ Lauren Seubert $^{1}$
}

Published online: 20 October 2015

(C) Psychonomic Society, Inc. 2015

\begin{abstract}
Item bank stratification has been shown to be an effective method for combating item overexposure in both uni- and multidimensional computer adaptive testing. However, item bank stratification cannot guarantee that items will not be overexposed - that is, exposed at a rate exceeding some prespecified threshold. In this article, we propose enhancing stratification for multidimensional computer adaptive tests by combining it with the item eligibility method, a technique for controlling the maximum exposure rate in computerized tests. The performance of the method was examined via a simulation study and compared to existing methods of item selection and exposure control. Also, for the first time, maximum likelihood (MLE) and expected a posteriori (EAP) estimation of examinee ability were compared side by side in a multidimensional computer adaptive test. The simulation suggested that the proposed method is effective in suppressing the maximum item exposure rate with very little loss of measurement accuracy and precision. As compared to MLE, EAP generates smaller mean squared errors of the ability estimates in all simulation conditions.
\end{abstract}

Keywords Multidimensional computerized adaptive test . Item selection · Exposure control $\cdot$ MLE $\cdot$ EAP

Computer adaptive tests (CATs) have been established as theoretically grounded and enormously practical assessment

Alan R. Huebner

Alan.Huebner.10@nd.edu

1 University of Notre Dame, 153 Hurley Hall, Notre Dame, IN 46556, USA

2 University of Minnesota, Minneapolis, MN, USA tools over the past several decades. Much of the early research in the field focused on CATs based on unidimensional item response theory (UIRT) models, but there has been increasing interest in multidimensional item response theory (MIRT) applications, such as in medical assessment (e.g., Norweg et al., 2011), patient report outcome measures (e.g., Zheng, Chang, \& Chang, 2013), and the Armed Services Vocational Aptitude Battery (ASVAB; e.g., Yao, Pommerich, \& Segall, 2014). Hereafter, we will use the acronyms UCAT and MCAT to refer to CATs based on UIRT and MIRT, respectively. Recent MCAT studies have addressed fundamental issues such as item selection (Mulder \& van der Linden, 2009; Segall, 1996; Veldkamp \& van der Linden, 2002; Wang \& Chang, 2011; Wang, Chang, \& Boughton, 2011; Yao, 2012), stopping rules (Wang, Chang, \& Boughton, 2013), and item exposure control (Finkelman, Nering, \& Roussos, 2009; Lee, Ip, \& Fuh, 2008). Lee et al. noted that most previous work on item exposure control had only addressed methods for UCAT, and they extended the item exposure method known as $a$-stratification (Chang \& Ying, 1999) to the MCAT case. Finkelman et al. proposed a method that controls the item exposure rates in an MCAT conditional on the examinees' ability levels. Since the Lee et al. method does not take into account examinee ability levels, it may be termed unconditional item exposure control, whereas the Finkelman et al. method would be referred to as conditional.

The present article focuses on unconditional MCAT item exposure control methods. Specifically, it expands the MCAT $a$-stratification (MSTR) method of Lee et al. (2008) by incorporating into it a method of maximum item exposure control. Leung, Chang, and Hau (2002) noted that $a$-stratification is effective in balancing the overall item exposure rates for UCAT, but it cannot guarantee that the exposure rate of any individual item will be held under a certain threshold. They proposed combining $a$ - 
stratification with the Sympson-Hetter (SH) method (Sympson \& Hetter, 1985) to control the maximum item exposure rate for UCAT. Our goal was similar for MCAT, but rather than use the SH method, which requires lengthy initial simulations, we used the item eligibility (IE) method (van der Linden \& Veldkamp, 2004, 2007), which can be implemented on the fly in an operational CAT. We also examined the effects on item exposure control arising from using maximum likelihood (ML) estimation of examinee ability versus expected a posteriori (EAP) estimation. A recent article by Wang (2015) compared these two methods generally for MIRT, but the present article is, to the best of our knowledge, the first to compare the two methods side by side in the context of MCAT.

Our discussion continues with the Method section, in which we review the MIRT model and the MSTR method of exposure control proposed by Lee et al. (2008), and describe our proposed addition of the IE method for maximum item exposure control, resulting in the MSTR-IE method. The next section recounts a simulation study designed to compare the performance of several item selection methods with and without exposure control under several testing conditions, and is followed by a section presenting the results. We conclude by summarizing our findings and suggesting topics for further exploration.

\section{Method}

\section{Multidimensional item response theory model}

MIRT models are based on the concept that subjects' responses to items are determined by a set of multiple latent abilities, or traits. The specific formulation of the model and the notation used are similar to those of Lee et al. (2008). The vector of $\boldsymbol{d}$ abilities possessed by each examinee is denoted as $\left\{\theta_{1}, \theta_{2}, \ldots, \theta_{d}\right\}$, where $d>1$ for the MIRT case. We let the random variable $U$ denote the response to a single dichotomous item, where values of 1 and 0 represent a correct and an incorrect response, respectively. Then, the vector $\boldsymbol{U}=\left(U_{1}, U_{2}\right.$, $\ldots, U_{j}$ ) is the collection of responses to $j$ items for a given examinee. The item response function is commonly seen as a two- or three-parameter logistic model, with the extra parameter being representative of the guessing probability. The form of the (compensatory) two-parameter MIRT model for item $j$ is given by

$P_{j}(\boldsymbol{\theta})=P_{j}(U=\mathbf{1} \mid \boldsymbol{\theta})=\frac{\exp \left[1.7 \alpha_{j}{ }^{\prime}\left(\theta-b_{j} 1\right)\right]}{\left\{1+\exp \left[1.7 \alpha_{j}\left(\theta-b_{j} 1\right)\right]\right\}}$.

Here, $\mathbf{1}$ is a $d \times 1$ dimensional vector of ones, $b_{j}$ is a scalar representing the item difficulty, and 1.7 is a scaling constant used in UIRT and MIRT models so that there is a high level of agreement between the logistic and probit link functions (Camilli, 1994; Lord, 1980). Also, $\alpha_{j}=\left\{a_{j 1}, a_{j 2}, \ldots, a_{j d}\right.$, $\}$ is the vector of item discrimination parameters for item $j$, where the elements in $\alpha_{j}$ correspond to the elements of $\boldsymbol{\theta}$. Although the methods reviewed and proposed are applicable to any $d>2$, for simplicity we will focus our presentation on the $d$ $=2$ case - that is, $\theta=\left\{\theta_{1}, \theta_{2}\right\}$.

\section{Ability estimation and item selection}

Given responses to a set of well-calibrated items, examinees may be scored via maximum likelihood estimation (MLE), expected a posteriori (EAP), or maximum a posteriori. In this study we compared the use of the first two methods in MCAT, and we review them here. The likelihood function of a vector containing responses from a single examinee to $j^{\prime}$ items is given by

$L(U \mid \theta)=\Pi_{j=1}^{j^{\prime}} P_{j}(\theta)^{U_{j}}\left(1-P_{j}(\theta)\right)^{1-U_{j}}$.

Then, taking partial derivatives of the log likelihood function with respect to $\boldsymbol{\theta}$ and setting this equal to zero, the ML ability estimate $\hat{\theta}^{M L E}$ is the solution to

$$
\left[\begin{array}{l}
\sum_{j=1}^{j^{\prime}}\left(\frac{\partial}{\partial \theta_{1}} \ln \frac{P_{j}(\theta)}{\left(1-P_{j}(\theta)\right)}\right)\left[u_{j}-P_{j}(\theta)\right] \\
\sum_{j=1}^{j^{\prime}}\left(\frac{\partial}{\partial \theta_{2}} \ln \frac{P_{j}(\theta)}{\left(1-P_{j}(\theta)\right)}\right)\left[u_{j}-P_{j}(\theta)\right]
\end{array}\right]=0 .
$$

The maximizing value of $\boldsymbol{\theta}$ may be obtained numerically by the Newton-Raphson method (Segall, 1996) or by the brute force method - that is, by plugging in a large number of $\left\{\theta_{1}, \theta_{2}\right\}$ values. Under the EAP estimation method, $\hat{\theta}^{E A P}$ is obtained using

$$
\hat{\theta}_{1}^{E A P}=\frac{\int \theta_{1} \int L(U \mid \theta) f(\theta) \partial \theta_{2} \partial \theta_{1}}{\iint L(U \mid \theta) f(\theta) \partial \theta_{2} \partial \theta_{1}} ; \quad \hat{\theta}_{2}^{E A P} \frac{\int \theta_{2} \int L(U \mid \theta) f(\theta) \partial \theta_{2} \partial \theta_{1}}{\iint L(U \mid \theta) f(\theta) \partial \theta_{2} \partial \theta_{1}},
$$

where $f(\theta)$ is the prior distribution of $\boldsymbol{\theta}$. Thus, $\hat{\theta}^{E A P}$ is an average over the posterior distribution of $\boldsymbol{\theta}$ (Wang, 2015).

In an MCAT, $\hat{\theta}^{M L E}$ or $\hat{\theta}^{E A P}$ is calculated after the administration of each item, and the current estimate is used to select the next item. Although there are various ways to select items in an MCAT (Wang \& Chang, 2011), the D-optimality method is one of the most common and was also the one used by Lee et al. (2008). Assuming that $j^{\prime}$ items have been administered, the next item $\left(j^{\prime}+1\right)$ is chosen so that it maximizes the quantity

$$
\begin{gathered}
D^{4}\left\{\sum_{j=1}^{j^{\prime}+1} a_{j 1}^{2} P_{j}(\hat{\theta})\left(1-P_{j}(\hat{\theta})\right) * \sum_{j=1}^{j^{\prime}+1} a_{j 2}^{2} P_{j}(\hat{\theta})\left(1-P_{j}(\hat{\theta})\right)\right. \\
\left.-\left(\sum_{j=1}^{j^{\prime}+1} a_{j 1} a_{j 2} P_{j}(\hat{\theta})\left(1-P_{j}(\hat{\theta})\right)\right)^{2}\right\} .
\end{gathered}
$$


Simpler criteria are available as well. As will be explained below, Lee et al. proposed a stratified item bank for MCAT; within each stratum at a given test stage, the method chooses the item with a difficulty parameter $b$ closest to the quantity

$\hat{b}=\frac{a_{j 1} \hat{\theta}_{1}+a_{j 2} \hat{\theta}_{2}}{a_{j 1}+a_{j 2}}$.

This is the two-dimensional version of a method used by Chang and Ying (1999) for stratification in a UCAT, which they reported was more efficient and performed similarly to selection via maximum information at $\hat{\theta}$.

\section{Item bank stratification for MCAT}

Item bank stratification as a means of exposure control for UCAT (Chang \& Ying, 1999) and its extensions (Chang, Qian, \& Ying, 2001; Chang \& van der Linden, 2003; Leung et al., 2002) has been well-documented and will not be reviewed here. We summarize the method proposed by Lee et al. (2008) for item bank stratification (MSTR) for MCAT, specifically focusing on the case of $d=2$ : (1) In the first step, the item bank is partitioned into $M$ levels - where items with the smallest $\left|a_{1}-a_{2}\right|$ are in the first stratum, items with the next smallest $\left|a_{1}-a_{2}\right|$ are in the second stratum, and so on - until items with the largest $\left|a_{1}-a_{2}\right|$ are in the stratum $M$. (2) Starting with the second stratum, each stratum is divided into two subdivisions, where substratum 1 contains items with $a_{1}>a_{2}$, and substratum 2 contains items for which $a_{1} \leq a_{2}$. (3) Then, the MCAT is divided into $M$ stages, where items for that stage are selected from the corresponding stratum. (4) Let $j_{m}$ be the total number of items administered for stratum $m$, and let $\sum_{m=1}^{J} j_{m}=J$, the total number of items on the test. Then, in Stratum 1 , select $j_{1}$ items using the $b$-matching rule described in the previous sections. (5) Then, for Stratum 2 and beyond, within a given stratum, items are selected from both subsets (alternating between subsets seems to be a reasonable strategy and was used for this study, as will be described below). Again, the $b$-match criterion is used when selecting items within a subset.

Lee et al. (2008) compared three different scenarios via a simulation study, selecting items using the D-optimality criterion with no exposure control, MSTR, and $b$-match used without stratification. Establishing the notation further, let $i$ $=1, \ldots, N$ be an index for the examinees, $\theta_{i 1}$ be the first element of the $\boldsymbol{\theta}$ vector of examinee $i$, and so on. Then, the quality of the recovery of the examinees' true $\boldsymbol{\theta}$ s was measured by the total bias and mean square error (MSE):

$$
\begin{aligned}
& \text { bias }=\frac{1}{N} \sum_{i=1}^{N}\left\{\left(\hat{\theta}_{i 1}-\theta_{i 1}\right)+\left(\hat{\theta}_{i 2}-\theta_{i 2}\right)\right\} \\
& M S E=\frac{1}{N} \sum_{i=1}^{N}\left\{\left(\hat{\theta}_{i 1}-\theta_{i 1}\right)^{2}+\left(\hat{\theta}_{i 2}-\theta_{i 2}\right)^{2}\right\} .
\end{aligned}
$$

In addition, the item exposure balance was quantified by using a $\chi^{2}$ statistic proposed by Chang and Ying (1999). Let $B$ be the total number of items in the bank and $b=1, \ldots, B$ be an index for the items. Also, the statistic

$e r_{b}=\frac{\text { number of timesitem } b \text { is exposed }}{N}$

indicates the exposure rate for a particular item; from an exposure control standpoint, $e r_{b}$ ideally would be equal for all $B$ items. This information is summarized for the entire bank using the statistic

$$
\chi^{2}=\sum_{b=1}^{B} \frac{\left(e r_{b}-e r_{b} / B\right)^{2}}{e r_{b} / B}
$$

Thus, smaller $\chi^{2}$ values indicate more uniform item exposure rates, whereas higher values imply that some items are exposed at higher rates than others. Lee et al. (2008) showed that in a variety of testing conditions the MSTR method had much lower $\chi^{2}$ values with slightly higher MSEs than the Doptimality criterion. Also, MSTR had slightly larger $\chi^{2}$ values and smaller MSEs than those yielded by $b$-match. Thus, it is reasonable to view MSTR as a "happy medium" between the D-optimality and $b$-match criteria, balancing item exposure control with measurement precision.

\section{Item bank stratification for MCAT with the IE method}

The issue of maximum item exposure control has lagged somewhat behind other areas of MCAT development, with the exception of Finkelman et al. (2009), who generalized the $\mathrm{SH}$ method to the multidimensional case. We wish to combine with item bank stratification a technique of maximum item exposure control such as the $\mathrm{SH}$ method, but $\mathrm{SH}$ has the drawback of requiring lengthy preliminary simulations in order to obtain the item exposure control parameters before operational testing may begin. Furthermore, this process may be especially burdensome when dealing with the increased computational complexity of the multidimensional case. Thus, we use the IE method of maximum item exposure control, an on-the-fly method that requires no preliminary simulations. Rather, the item exposure control parameters are updated after each examinee has been administered the exam. To review IE, we let $K_{b}{ }^{\left(i^{\prime}\right)}$ be the exposure control parameter for item $b$ after examinee $i^{\prime}$ has taken the exam, $r^{\max }$ the predetermined maximum desired item exposure rate, $A_{b}$ is the event that item $b$ is administered, and $P\left(1 \ldots i^{\prime}\right)\left(A_{b}\right)$ the probability of $A_{b}$ computed from examinees $1, \ldots, i^{\prime}$. For example, if examinee $i$ is the 100th examinee to take the exam in a certain timeframe and item $b$ was administered to ten of them, then $P^{\left(1 \ldots i^{\prime}\right)}\left(A_{b}\right)=10 / 100$. Then, $K_{b}{ }^{(i+1)}$, the item 
Table 1 Summary of factors varied in the simulation study

\begin{tabular}{ll}
\hline Factor & Levels \\
\hline Test length & 20 \\
& 30 \\
& 60 \\
$\rho$ & .30 \\
& .60 \\
$\theta$ estimation & .80 \\
& MLE \\
Selection method/exposure control & EAP(informative prior) \\
& EAP(noninformative prior) \\
& MSTR \\
& MSTR-IE \\
\hline
\end{tabular}

exposure parameter for item $b$ for examinee $\left(i^{\prime}+1\right)$, is given by

$k_{b}^{\left(i^{\prime}+1\right)}=\left\{\begin{array}{c}1 \quad \text { if } P^{\left(1 \ldots i^{\prime}\right)}\left(A_{b}\right) / K_{b}^{\left(i^{\prime}\right)} \leq r^{\max } \\ \frac{r^{\max } k_{b}^{\left(i^{\prime}\right)}}{P^{\left(1 \ldots i^{\prime}\right)}\left(A_{b}\right)} \text { if } P^{\left(1 \ldots i^{\prime}\right)}\left(A_{b}\right) / K_{b}^{\left(i^{\prime}\right)}>r^{\max }\end{array}\right.$.

Thus, IE takes into account $r^{\max }$ and $P\left(1 \ldots i^{\prime}\right)\left(A_{b}\right)$ in a probabilistic manner, so that item exposure rates do not exceed $r^{\max }$ and the order of the items is not predictable (Barrada, Abad, \& Veldkamp, 2009).

We now describe our proposed method for integrating item bank stratification for MCAT with IE, resulting in the enhanced method MSTR-IE. Steps 1 through 3 are identical to those for the MSTR method listed in the previous section, so we begin with the MSTR-IE at Step 4:

(4) In Stratum 1, select items as follows. For examinee $i^{\prime}$, use the $b$-match rule to identify an item for potential administration, notated $b^{\prime}$. Generate $U$, a Uniform $(0,1)$ random number, and administer item $b^{\prime}$ if $U \leq K_{b^{\prime}}^{\left(i^{\prime}\right)}$. If $U>K_{b^{\prime}}^{\left(i^{\prime}\right)}$, then item $b^{\prime}$ is no longer eligible for administration to examinee $i^{\prime}$, and a new item is selected using $b$-match. The process is continued until $j_{1}$ items have been administered from Stratum 1.

(5) For Stratum 2 and higher, continue the process of selecting items using $b$-match generating $U \mathrm{~s}$ and comparing them to the item exposure control parameters. Alternate between subsets in a given stratum.

(6) After the completion of the test for examinee $i^{\prime}$, update the exposure control parameters by calculating $K_{b}^{\left(i^{\prime}+1\right)}$ for all bank items $b=1, \ldots B$, and use these for examinee $\left(i^{\prime}+1\right)$.

\section{Simulation study}

A simulation study was performed to examine the ability of the proposed MSTR-IE exposure control method to prevent item exposure rates from exceeding $r^{\max }$ while maintaining accurate and precise measurement of examinee ability. Several factors were manipulated to mimic various testing conditions for a realistic MCAT measuring $d=2$ latent abilities. These factors included test length $(20,30$, and 60 items), the correlation between the latent abilities $\theta_{1}$ and $\theta_{2}(\rho=.30$, .60 , and .80), the $\theta$ estimation method (EAP with both informative and noninformative prior distributions, as will be explained below, and MLE), and the item selection/exposure control method used (D-optimality, MSTR, and MSTR-IE). The test lengths and values of $\rho$ included those used in the simulation study performed by Lee et al. (2008). Table 1 summarizes the manipulated factors and their levels. Four factors, each having three levels, resulted in $3 \times 3 \times 3 \times 3=81$ total conditions run. The value of $r^{\max }$ was set to .20 , a commonly used maximum exposure rate (Leung et al., 2002). The results in each condition were evaluated using the bias, MSE, and $\chi^{2}$ statistics described above, as well as the observed maximum item exposure rate (MIER) and the proportion of items with exposure rates over $r^{\max }$ (OEXP). For example, if a particular testing condition with $r^{\max }=.20$ yields MIER $=.25$ and OEXP $=.05$, this means that the most-administered item

Table 2 Summary of item strata used at each state of the tests

\begin{tabular}{llll}
\hline Stratum & Test Stages for Lengths $J=20 / 30 / 60$ & $\alpha_{1}$ & $\alpha_{2}$ \\
\hline 1 & $(4-5) /(4-7) /(4-15)$ & $0.70(0.32)$ & $0.70(0.33)$ \\
2 & $(6-10) /(8-14) /(16-30)$ & $0.82(0.29) / 0.52(0.31)$ & $0.53(0.30) / 0.81(0.29)$ \\
3 & $(11-15) /(15-22) /(31-45)$ & $0.89(0.22) / 0.37(0.23)$ & $0.38(0.21) / 0.90(0.23)$ \\
4 & $(16-20) /(23-30) /(45-60)$ & $1.07(0.15) / 0.18(0.12)$ & $0.22(0.14) / 1.03(0.16)$ \\
\hline
\end{tabular}

For Strata 2-4, results are reported as the means (standard deviations) for substratum 1/substratum 2. 
Table 3 Simulation study results for all conditions

\begin{tabular}{|c|c|c|c|c|c|}
\hline Length & Method & Statistic & MLE & EAP(Inf) & EAP(flat) \\
\hline \multirow[t]{15}{*}{20} & \multirow[t]{5}{*}{ D-optim } & Bias & $-0.010 /-0.008 /-0.002$ & $0.003 /-0.001 /-0.007$ & $0.002 /-0.006 /-0.004$ \\
\hline & & $M S E$ & $0.416 / 0.433 / 0.431$ & $0.263 / 0.261 / 0.255$ & $0.337 / 0.344 / 0.347$ \\
\hline & & $\chi^{2}$ & $96.67 / 93.21 / 91.67$ & $109.07 / 100.99 / 100.86$ & $102.15 / 98.04 / 94.96$ \\
\hline & & MIER & $0.62 / 0.59 / 0.59$ & $0.71 / 0.66 / 0.66$ & $0.62 / 0.62 / 0.60$ \\
\hline & & OEXP & $0.07 / 0.07 / 0.07$ & $0.07 / 0.07 / 0.07$ & $0.08 / 0.07 / 0.07$ \\
\hline & \multirow[t]{5}{*}{ MSTR } & Bias & $-0.007 / 0.006 / 0.002$ & $0.007 / 0.011 / 0.009$ & $0.007 /-0.008 /-0.001$ \\
\hline & & $M S E$ & $0.838 / 0.832 / 0.827$ & $0.429 / 0.401 / 0.371$ & $0.620 / 0.623 / 0.616$ \\
\hline & & $\chi^{2}$ & $7.98 / 8.79 / 9.81$ & $5.90 / 5.58 / 5.60$ & $5.01 / 6.30 / 6.82$ \\
\hline & & MIER & $0.25 / 0.25 / 0.26$ & $0.13 / 0.13 / 0.15$ & $0.16 / 0.17 / 0.18$ \\
\hline & & OEXP & $0.00 / 0.00 / 0.00$ & $0.00 / 0.00 / 0.00$ & $0.00 / 0.00 / 0.00$ \\
\hline & \multirow[t]{5}{*}{ MSTR-IE } & Bias & $0.000 /-0.001 /-0.007$ & $0.013 / 0.008 / 0.010$ & $-0.005 /-0.011 / 0.006$ \\
\hline & & MSE & $0.816 / 0.838 / 0.826$ & $0.422 / 0.388 / 0.355$ & $0.619 / 0.635 / 0.61$ \\
\hline & & $\chi^{2}$ & $7.26 / 8.07 / 8.39$ & $5.66 / 5.54 / 5.69$ & $5.18 / 5.98 / 7.14$ \\
\hline & & MIER & $0.20 / 0.20 / 0.20$ & $0.13 / 0.13 / 0.13$ & $0.16 / 0.17 / 0.18$ \\
\hline & & OEXP & $0.00 / 0.00 / 0.00$ & $0.00 / 0.00 / 0.00$ & $0.00 / 0.00 / 0.00$ \\
\hline \multirow[t]{15}{*}{30} & \multirow[t]{5}{*}{ D-optim } & Bias & $-0.001 /-0.005 /-0.004$ & $0.002 / 0.002 /-0.001$ & $-0.008 /-0.005 /-0.004$ \\
\hline & & $M S E$ & $0.263 / 0.261 / 0.285$ & $0.193 / 0.189 / 0.187$ & $0.221 / 0.232 / 0.245$ \\
\hline & & $\chi^{2}$ & $104.09 / 102.88 / 100.61$ & $111.89 / 106.22 / 103.82$ & $105.02 / 103.24 / 101.08$ \\
\hline & & MIER & $0.65 / 0.65 / 0.65$ & $0.73 / 0.71 / 0.69$ & $0.65 / 0.65 / 0.64$ \\
\hline & & OEXP & $0.12 / 0.12 / 0.12$ & $0.12 / 0.12 / 0.12$ & $0.12 / 0.12 / 0.11$ \\
\hline & \multirow[t]{5}{*}{ MSTR } & Bias & $-0.002 /-0.007 /-0.002$ & $0.000 /-0.002 /-0.003$ & $-0.002 / 0.006 /-0.007$ \\
\hline & & $M S E$ & $0.486 / 0.499 / 0.487$ & $0.304 / 0.292 / 0.283$ & $0.419 / 0.417 / 0.406$ \\
\hline & & $\chi^{2}$ & $7.58 / 8.78 / 9.53$ & $7.04 / 6.96 / 7.38$ & $6.04 / 7.45 / 7.85$ \\
\hline & & MIER & $0.26 / 0.26 / 0.26$ & $0.18 / 0.17 / 0.18$ & $0.18 / 0.19 / 0.18$ \\
\hline & & OEXP & $0.00 / 0.00 / 0.00$ & $0.00 / 0.00 / 0.00$ & $0.00 / 0.00 / 0.00$ \\
\hline & \multirow[t]{5}{*}{ MSTR-IE } & Bias & $0.00 /-0.008 / 0.002$ & $0.001 / 0.003 /-0.004$ & $0.006 /-0.007 / 0.002$ \\
\hline & & $M S E$ & $0.480 / 0.503 / 0.488$ & $0.311 / 0.283 / 0.28$ & $0.395 / 0.412 / 0.409$ \\
\hline & & $\chi^{2}$ & $7.21 / 8.22 / 8.91$ & $7.25 / 6.88 / 7.15$ & $6.18 / 7.18 / 8.23$ \\
\hline & & MIER & $0.20 / 0.20 / 0.20$ & $0.19 / 0.18 / 0.17$ & $0.18 / 0.19 / 0.19$ \\
\hline & & OEXP & $0.00 / 0.00 / 0.00$ & $0.00 / 0.00 / 0.00$ & $0.00 / 0.00 / 0.00$ \\
\hline \multirow[t]{15}{*}{60} & \multirow[t]{5}{*}{ D-optim } & Bias & $-0.003 /-0.002 /-0.003$ & $0.000 /-0.001 /-0.009$ & $0.000 / 0.002 / 0.003$ \\
\hline & & $M S E$ & $0.135 / 0.143 / 0.150$ & $0.117 / 0.116 / 0.115$ & $0.130 / 0.132 / 0.135$ \\
\hline & & $\chi^{2}$ & $108.29 / 103.02 / 100.86$ & $110.41 / 105.50 / 101.95$ & $107.91 / 102.14 / 99.76$ \\
\hline & & MIER & $0.75 / 0.73 / 0.72$ & $0.79 / 0.77 / 0.75$ & $0.76 / 0.72 / 0.72$ \\
\hline & & OEXP & $0.27 / 0.26 / 0.26$ & $0.26 / 0.27 / 0.26$ & $0.26 / 0.27 / 0.27$ \\
\hline & \multirow[t]{5}{*}{ MSTR } & Bias & $-0.004 / 0.002 / 0.003$ & $0.000 / 0.006 /-0.007$ & $-0.002 /-0.009 /-0.004$ \\
\hline & & $M S E$ & $0.233 / 0.240 / 0.233$ & $0.182 / 0.179 / 0.170$ & $0.216 / 0.218 / 0.219$ \\
\hline & & $\chi^{2}$ & $5.12 / 5.56 / 5.87$ & $6.60 / 5.30 / 5.25$ & $5.81 / 5.37 / 6.11$ \\
\hline & & MIER & $0.28 / 0.28 / 0.30$ & $0.31 / 0.28 / 0.27$ & $0.28 / 0.26 / 0.27$ \\
\hline & & OEXP & $0.02 / 0.02 / 0.03$ & $0.05 / 0.02 / 0.03$ & $0.03 / 0.02 / 0.04$ \\
\hline & \multirow[t]{5}{*}{ MSTR-IE } & Bias & $0.001 / 0.001 / 0.006$ & $0.006 /-0.001 /-0.001$ & $0.000 /-0.006 / 0.001$ \\
\hline & & $M S E$ & $0.237 / 0.247 / 0.244$ & $0.179 / 0.177 / 0.172$ & $0.215 / 0.221 / 0.218$ \\
\hline & & $\chi^{2}$ & $4.51 / 5.09 / 5.38$ & $5.65 / 4.93 / 4.98$ & $4.68 / 4.92 / 5.58$ \\
\hline & & MIER & $0.20 / 0.20 / 0.20$ & $0.20 / 0.20 / 0.20$ & $0.20 / 0.20 / 0.20$ \\
\hline & & OEXP & $0.00 / 0.01 / 0.01$ & $0.02 / 0.01 / 0.01$ & $0.01 / 0.01 / 0.02$ \\
\hline
\end{tabular}

In each cell results are shown for $\rho=.30 / .60 / .80 . M S E=$ mean squared error; MIER = maximum item exposure rate; OEXP = proportion of items with exposure rates exceeding $r^{\max } ; \mathrm{MLE}=$ maximum likelihood; $\mathrm{EAP}(\mathrm{inf})=$ expected a posteriori with informative prior; $\mathrm{EAP}(\mathrm{flat})=\operatorname{expected}$ a posteriori with flat prior 
was seen by $25 \%$ of the examinees, and $5 \%$ of the items were exposed to at least $20 \%$ of the examinees.

All conditions used a simulated item bank consisting of 480 items with parameters drawn from the following distributions: $\quad a_{1} \sim \operatorname{Uniform}(0,1.3), \quad a_{2} \sim \operatorname{Uniform}(0,1.3), \quad$ and $b \sim$ Uniform $(-1.3,1.3)$. These settings are the same as were used in several recent MCAT studies (Finkelman et al., 2009; van der Linden \& Veldkamp, 2007; Wang \& Chang, 2011) and are meant to reflect the values found in operational tests; in contrast, Lee et al. (2008) constructed an item bank for their simulation study in which the $a_{1}$ and $a_{2}$ parameters were systematically manipulated. In each condition, $N=5,000$ examinees were administered exams, with $\boldsymbol{\theta}$ s drawn from the bivariate normal distribution,

$\left(\begin{array}{l}\theta_{1} \\ \theta_{2}\end{array}\right) \sim$ bivariate normal $\left(\left(\begin{array}{l}0 \\ 0\end{array}\right),\left[\begin{array}{ll}1 & \rho \\ \rho & 1\end{array}\right]\right)$.

MLE with D-optimality

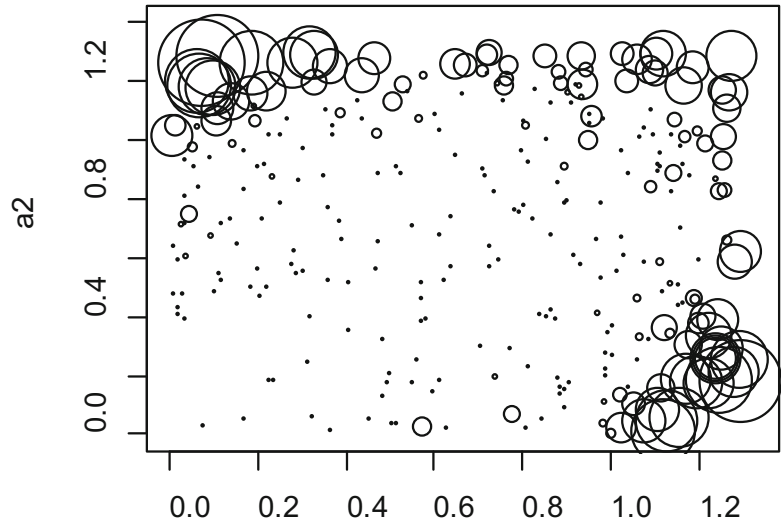

a1

MLE with MSTR-IE

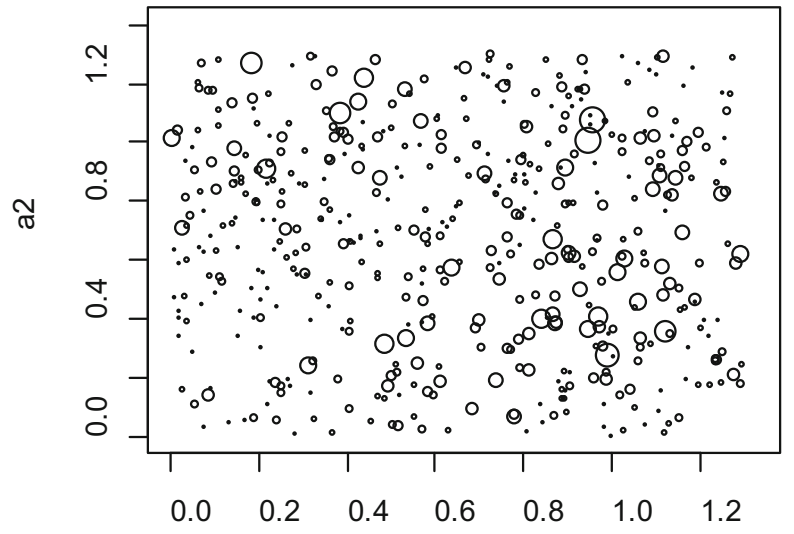

a1
Both EAP and MLE estimation were conducted using the brute force method. Specifically, for both $\theta_{1}$ and $\theta_{2}$, vectors were created ranging from -3 to 3 in steps of 0.1 , resulting in 61 values along each dimension. Then, each $\theta_{1}$ value was paired with the entire $\theta_{2}$ vector, resulting in $61^{2}=3,721$ $\left\{\theta_{1}, \theta_{2}\right\}$ pairs. The particular $\left\{\theta_{1}, \theta_{2}\right\}$ pair that maximized the $\log$ likelihood function $L(U \mid \theta)$ was taken to be $\hat{\theta}^{M L E}$, and these values were used as quadrature nodes in the integration to obtain $\hat{\theta}^{E A P}$. Following Wang (2015), the informative and noninformative (i.e., flat) prior distributions had diagonal covariance matrices with $1 \mathrm{~s}$ and $10 \mathrm{~s}$ on the diagonals, respectively, and both used a zero mean vector. It is expected that EAP with a noninformative prior will behavior more similarly to MLE than will EAP with an informative prior. Each exam started with three randomly drawn items to establish a preliminary estimate of $\boldsymbol{\theta}$, and for the MSTR and MSTR-IE methods, the item bank was divided into four strata, each

EAP (inf) with D-optima

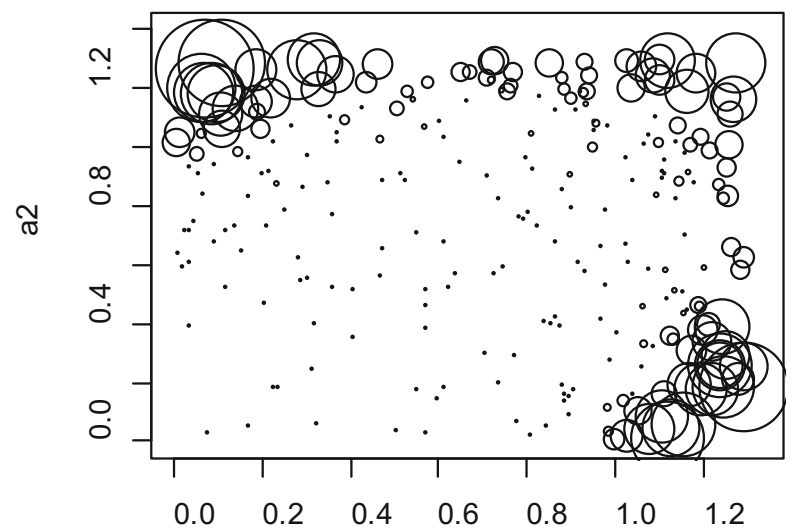

a1

EAP (inf) with MSTR -IE

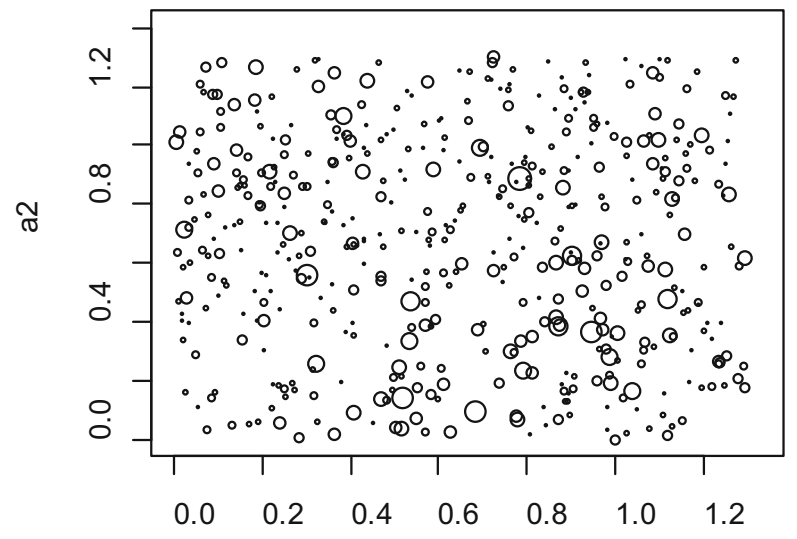

a1

Fig. 1 Plots of the discrimination parameters for exposed items. The areas of the circles are proportional to the exposure rates of the items. All plots are for conditions with $J=30$ and $\rho=.30$ 
having 120 items. The second column of Table 2 shows the stratum used at a given stage of the test under all test lengths. For example, items were selected from item bank Stratum 2 at Stages 6-10 for the 20-item tests, Stages 8-14 for the 30-item tests, and Stages 16-30 for the 60-item tests, and so on. Also, the third and fourth columns of Table 2 give summary statistics for the item parameters $a_{1}$ and $a_{2}$, respectively, in each stratum. Specifically, the means are reported with standard deviations (SDs) in parentheses, and for Strata 2-4 statistics are reported for both subsections. For example, in subsection 1 of Stratum 2, the mean $a_{1}$ was $0.82(S D=0.29)$, and in subsection 2 of Stratum 1 , the mean $a_{1}$ was $0.52(S D=0.31)$, and so on.

\section{Results}

All simulation scripts, analyses, and plots were done using the $\mathrm{R}$ statistical software environment ( $\mathrm{R}$ Development Core Team, 2013). Table 3 presents the results from the simulation study for all conditions. First, we see that selecting items via D-optimality with no exposure control yields very large $\chi^{2}$ values, and the MIER is at least $59 \%$ for MLE and both versions of EAP under all test lengths and correlations. Also, OEXP was at least $7 \%, 12 \%$, and $26 \%$ under D-optimality for the 20-, 30-, and 60-item test lengths, respectively. Thus, the problem of item overexposure increased with the test lengths, which is not surprising. Table 3 also shows that MSTR and MSTR-IE greatly reduced the values of $\chi^{2}$ relative to D-optimality, though there was not a large difference between MSTR and MSTR-IE for a given test length and $\rho$ value. In the $J=20$ block of Table 3, we see that the MSTR method alone eliminated almost all instances of item overexposure, except for a small number of items that caused the MIER to be .26 or .25 under MLE estimation. (For these cases, the OEXP was 0.00 due to rounding to two decimal places.) A similar situation is seen for the $J=30$ conditions in the table. The utility of the MSTR-IE method becomes more apparent when considering the longer tests. For $J=60$, MSTR was not able to keep the MIER below .20, but, as expected, MSTR-IE held MIER at or very near .20. The MSTR-IE is capable of suppressing the MIER to be very near $r^{\max }$, but not strictly equal or less. This is because, as Barrada et al. (2009) explained, the IE is probabilistic in nature, and the $r^{\max }$ threshold may be violated for the most informative items.

Figure 1 provides a more intuitive depiction the item exposure rates under different selection criteria. As a representative selection, we focus on MLE and EAP(inf) using D-optimality and MSTR-IE with $J=30$ and $\rho=.30$. Each item in the bank is plotted as a circle in a two-dimensional space with the discrimination parameters $\left\{a_{1}, a_{2}\right\}$ along the axes, and the areas of the circles are proportional to the exposure rates of

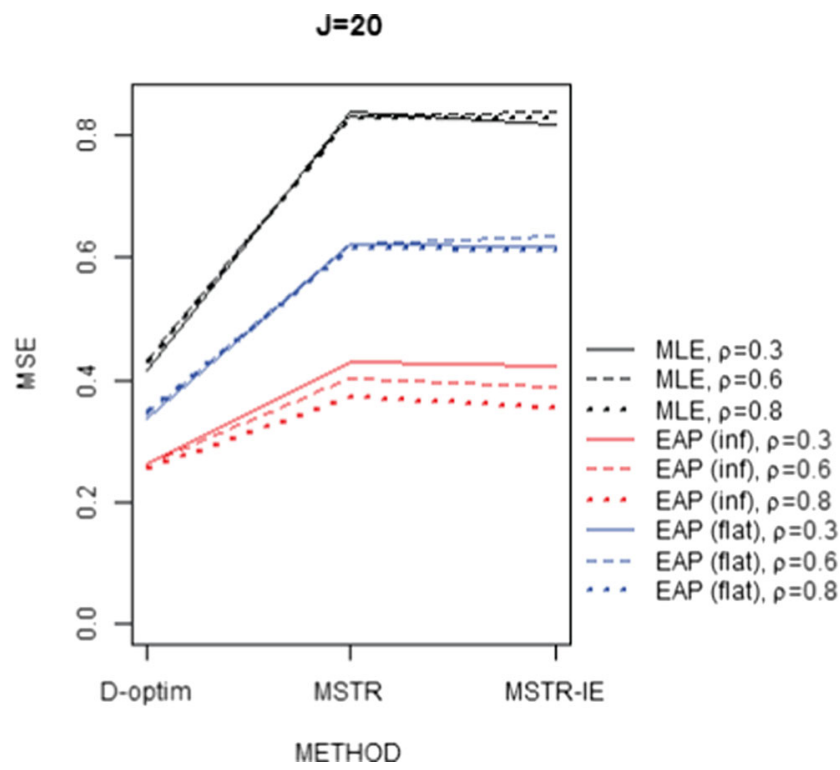

Fig. $2 M S E$ s for all $J=20$ conditions by estimation method, selection/ exposure control method, and correlation

the items. Items not exposed to any examinee appear as points with no area. For the conditions using D-optimality, there are very large circles - that is, high exposure rates - for items having a value of at least 1 at one or both discrimination parameters. In contrast, the circles are much more uniform in size in the plots for testing conditions using MSTR-IE. This general pattern was consistent under all testing conditions, and for a given $J$ and $\rho$, the MSTR and MSTR-IE methods produced very similar plots.

Next, we consider the accuracy and precision of the $\boldsymbol{\theta}$ estimates; it can be seen in Table 3 that all of the methods are essentially unbiased, on average. It is more interesting to

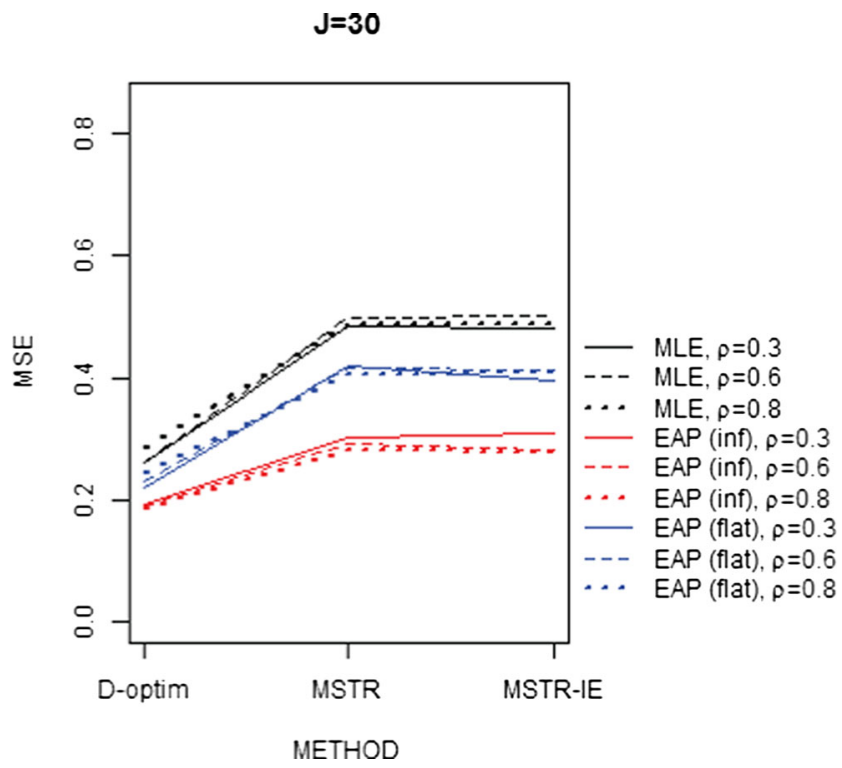

Fig. 3 MSEs for all $J=30$ conditions by estimation method, selection/ exposure control method, and correlation 


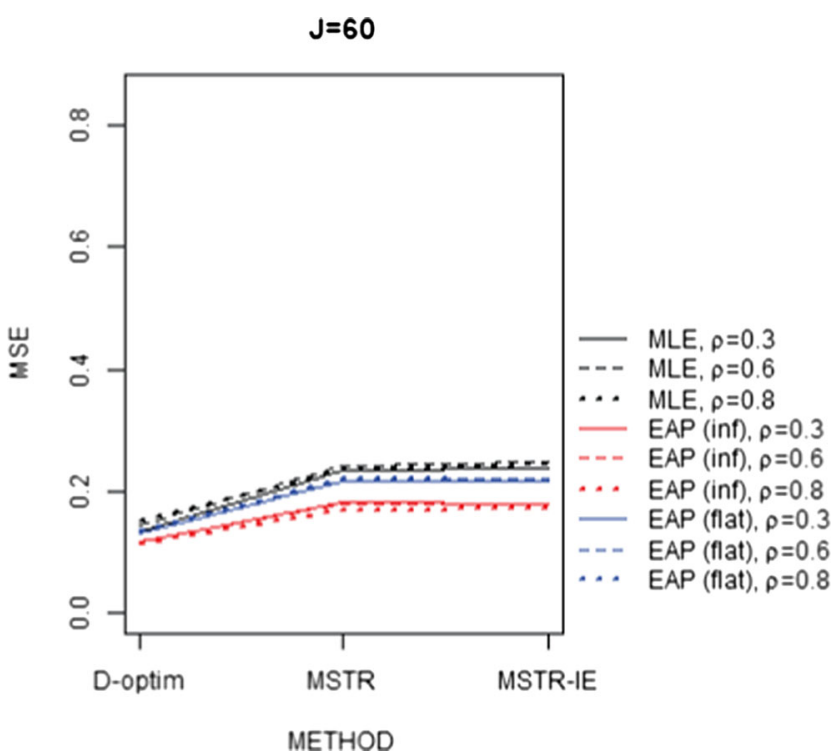

Fig. $4 M S E$ s for all $J=60$ conditions by estimation method, selection/ exposure control method, and correlation

\section{MLE with D-optim}

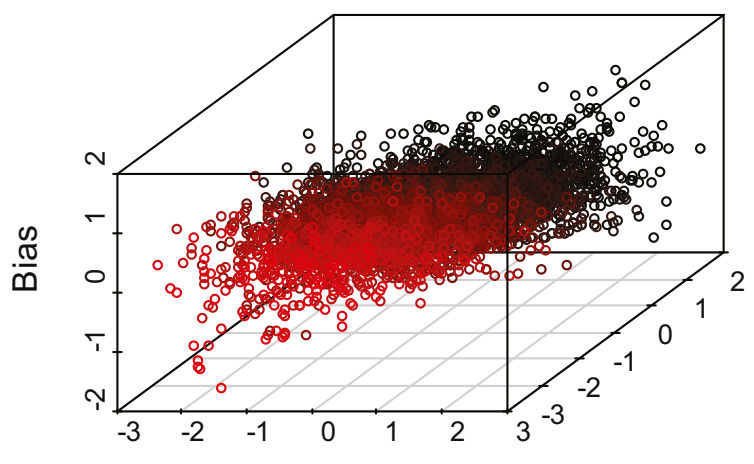

$\theta 1$

MLE with MSTR-IE

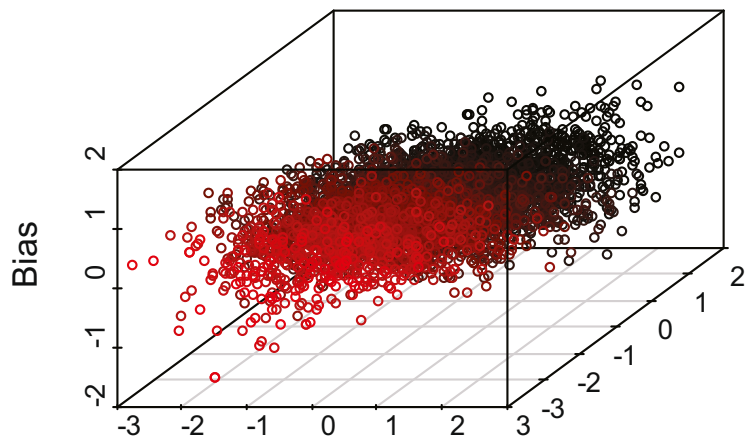

$\theta 1$ examine the interplay between the $M S E$ and estimation method; Figs. 2, 3, and 4 display the MSEs by estimation method, selection/exposure control method, and correlation for each test length. Thus, each of these figures represents 27 testing conditions. First, it can easily be seen that the MSE is smaller for EAP than for MLE in all conditions, and that EAP with an informative prior has smaller MSEs than EAP with a flat prior. This is because adding a prior in EAP helps to bring down the posterior variance of $\boldsymbol{\theta}$ estimates, resulting in a lower MSE. As compared to a flat prior, the more informative prior adds higher information to help locate $\boldsymbol{\theta}$ estimates in the multidimensional space, and thus it produces a smaller MSE. The differences between the MSEs yielded by the estimation methods decrease as the test length increases. For longer tests, the test information plays a dominant role as compared to the prior, and thus the difference between MLE and EAP diminishes (Wang, 2015). Also, MSE increases in all conditions moving from selecting items via D-optimality to MSTR or MSTR-IE. The nature of this increase is noteworthy; in all three figures, the lines for the MLE are steeper than those for EAP with an informative prior, suggesting an interaction

\section{EAP (inf) with D-optim}

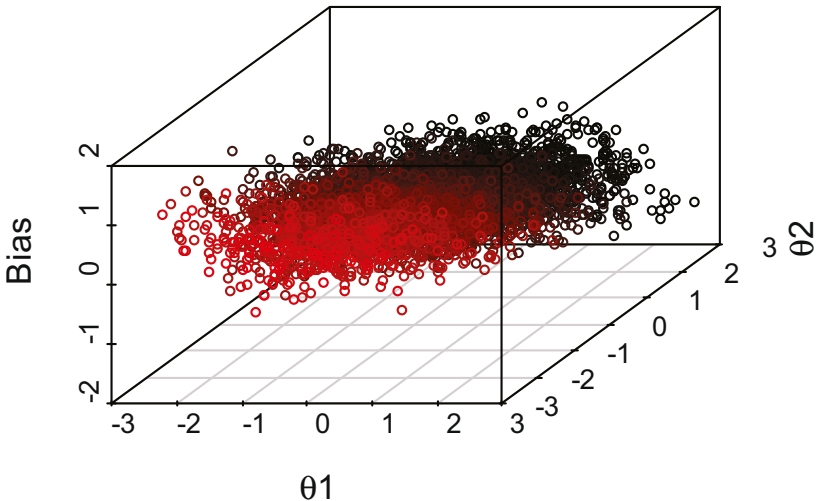

EAP (inf) with MSTR-IE

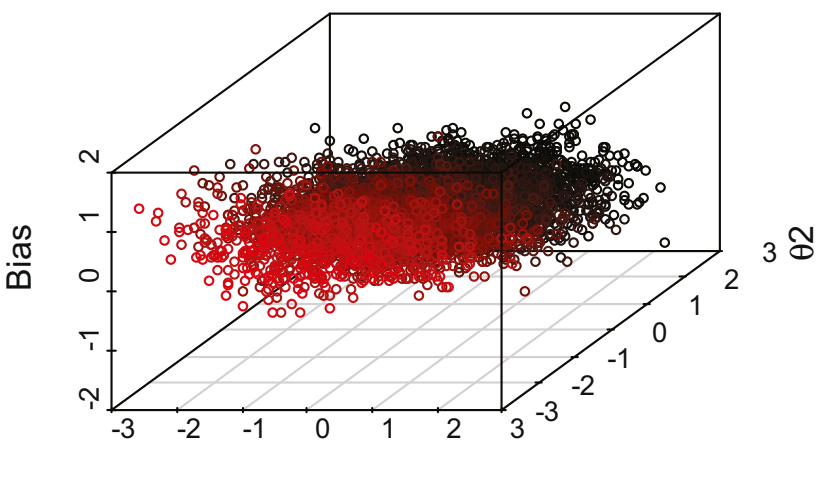

$\theta 1$

Fig. 5 Three-dimensional scatterplots for bias, where the $x, y$, and $z$ axes represent the true $\theta_{1}$ value, the true $\theta_{2}$ value, and the bias, respectively, for $J=$ 30 and $\rho=.30$ 
between the methods of item selection and examinee ability estimation. Specifically, the MSE increases more steeply when moving from D-optimality to MSTR/MSTR-IE when using MLE than for EAP(informative). This effect appears smallest for $J=60$ and largest for $J=20$. Such a phenomenon is again due to the inclusion of the prior in EAP. However, there is a smaller difference between the slopes for MLE and EAP with a flat prior. In some conditions, the rates of increase in the MSE moving from D-optimality to a stratified method are nearly equal for those two estimation methods.

Also, Figs. 2, 3 and 4 distinguish between different correlation levels via the use of solid, dashed, and dotted lines. It can be seen that the trends described above hold for all three correlation levels, and the differences in the MSEs between correlation levels for estimation and selection methods decrease as the test length increases. Apart from these observations, the effect of latent trait correlation on MSEs is inconclusive. The largest differences in MSEs between correlation levels occurs for EAP with an informative prior for $J=20$, with a larger correlation being associated with a smaller MSE, but this trend does not hold for other testing conditions. In general, our results seem to be consistent with the findings of Lee et al. (2008), whose study did not show large or consistent differences in bias, MSE, or $\chi^{2}$ over various levels of correlation.

Although Table 3 presents overall bias and MSE statistics, we believe it is also beneficial for practitioners to see results disaggregated across the ability ranges. Figures 5 and 6 display three-dimensional scatterplots for the bias and MSE for both MLE and EAP(inf) estimation under D-optimality and MSTR-IE for $J=30$ and $\rho=.30$. Each point represents an individual examinee, and the $x, y$, and $z$ axes represent the true $\theta_{1}$ value, the true $\theta_{2}$ value, and the bias or MSE, respectively, for that individual. The colors enhance the three-dimensional aspect by indicating location on the $\theta_{2}$ axis; specifically, the points with $\theta_{2}$ values closer to -3.0 appear red, and those with $\theta_{2}$ values closer to 3.0 appear black. In Fig. 5, we see in all four panels that the individuals with extreme $\theta_{1}$ and/or $\theta_{2}$ values tend to have more biased estimates. The pattern is similar but less clear for the MSE plots displayed in Fig. 6. For the sake of brevity, it is inconvenient to display such plots for all conditions; the simulated data (in the form of $\mathrm{R}$

\section{MLE with D-optim}

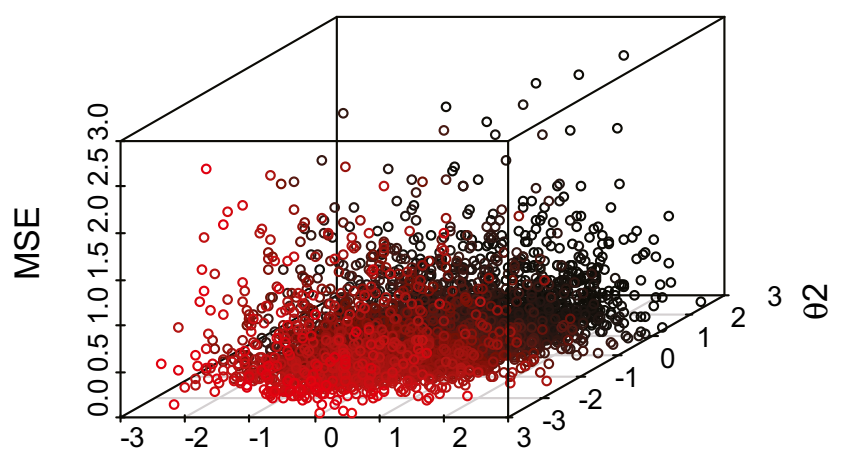

$\theta 1$

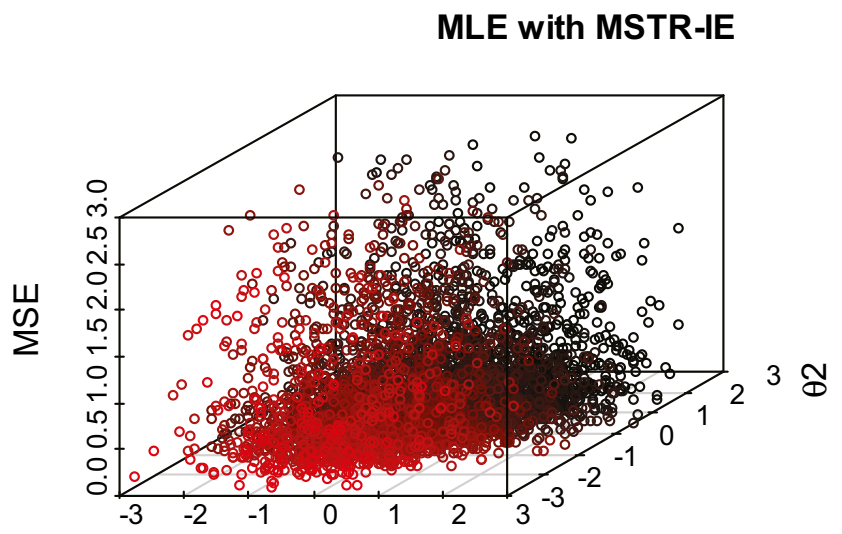

$\theta 1$

\section{EAP (inf) with D-optim}

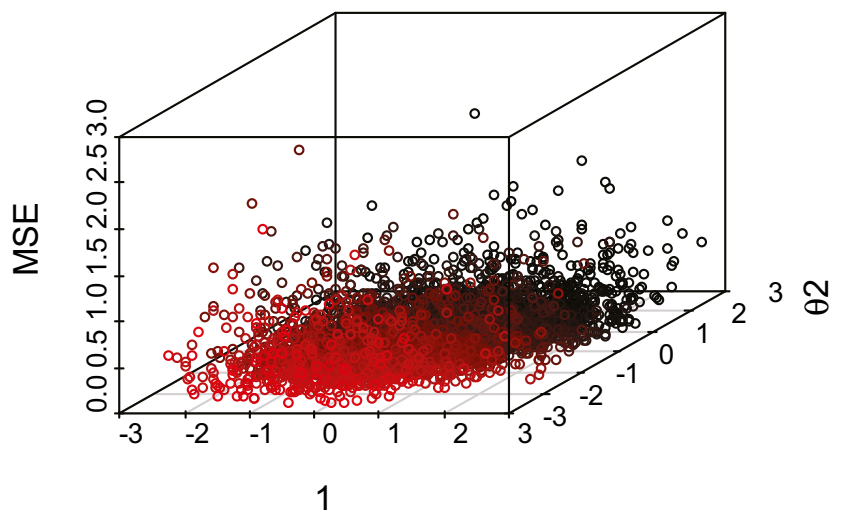

EAP (inf) with MSTR-IE

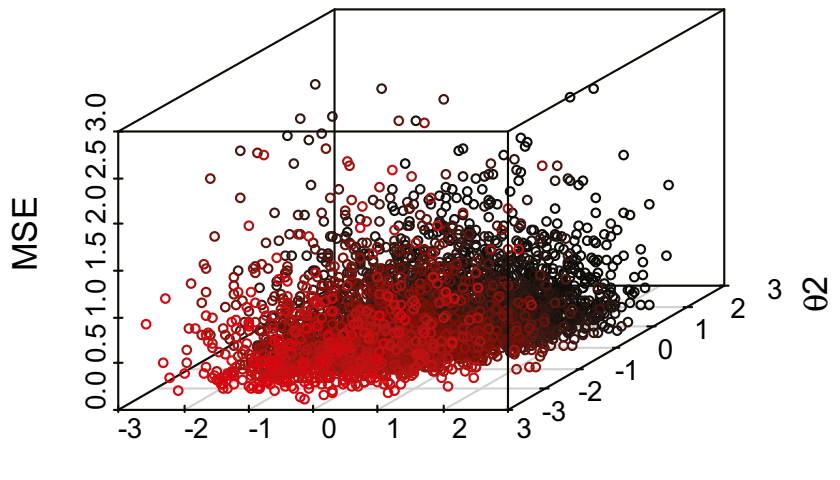

$\theta 1$

Fig. 6 Three-dimensional scatterplots for bias, where the $x, y$, and $z$ axes represent the true $\theta_{1}$ value, the true $\theta_{2}$ value, and the $M S E$, respectively, for $J=$ 30 and $\rho=.30$ 
workspaces) and an R script to generate the plots are available from the corresponding author.

\section{Summary and discussion}

In this article, we proposed the MSTR-IE method of item exposure control for MCAT. It is an enhancement of the MSTR method proposed by Lee et al. (2008) and was shown via a simulation study to effectively suppress the MIER to be very close to $r^{\max }$, the target maximum item exposure rate, with virtually no increase in bias or MSE. The MSTR-IE was shown to be more relevant for longer test lengths, because for $J=20$ and 30 the MSTR alone may be capable of preventing exposure rates from exceeding $r^{\max }$ for all but a few items. In addition, MLE and EAP estimation were compared side by side for the first time in an MCAT setting, and this revealed an interaction between the method of item selection/exposure control and ability estimation on MSE. Specifically, the increase in MSE resulting from using a stratified item bank was greater under MLE than under EAP estimation. Thus, if researchers are interested in stratifying an operational MCAT item bank, the use of EAP estimation should be considered. When using EAP, the difference between an informative and a flat prior diminishes when the correlation between dimensions increases and/or when the test length increases. In practice, for a reasonably long test (e.g., test length $=60$ ), both priors can be selected without appreciable difference; for a relatively short test, the informative prior is preferred if practitioners have some knowledge about the population distribution of $\boldsymbol{\theta}$.

There are some limitations to the study, since no single article can investigate the myriad of possible variations on testing conditions. One factor we did not vary was the item bank, and it is possible that the differences between the methods may be enhanced or diminished when they are used with different item banks. Another issue is the use of the IE method within the MSTR-IE. The IE method is an example of on-the-fly exposure control, in which the exposure control parameters are continually updated as examinees take the test. Although this type of approach has advantages over the more traditional SH method, it is possible that an on-the-fly approach may be deemed inappropriate or infeasible for a given operational program. In such cases, the SH method may be used to control the maximum exposure rate within MSTR. The results of Barrada et al. (2009) suggest that for UCAT, the SH method performs similarly to IE, though it is slightly inferior with respect to MIER and OEXP.

There are several avenues for further research. Currently there are no guidelines on exactly how to determine the number of strata required for MSTR or MSTR-IE in an MCAT. Lee et al. (2008) provided the general advice that the more the $\left|a_{1}-a_{2}\right|$ values vary, the greater the number of strata needed.
Barrada, Abad, and Olea (2014) proposed a method for finding the optimal number of strata in a UCAT item bank; a future study may explore the feasibility of extending this method to the MCAT case. Also, when implementing a stratified item bank in an MCAT, some researchers and practitioners may wish to have greater control over the balance between item exposure control and MSE yielded by the selection/exposure control method. In particular, it may be desired to obtain a lower MSE than is currently yielded by the MSTR or MSTR-IE method, in exchange for a higher $\chi^{2}$. A future study may address the method of item selection within each stratum. For example, the restrictive progressive and restrictive threshold methods were proposed by Wang, Chang, and Huebner (2011) for balancing item exposure versus measurement accuracy in the context of cognitive diagnostic CAT. However, these are flexible methods that are applicable to selecting items within a stratum in an MCAT setting and may prove able to strike a balance between D-optimality (lowest MSE, very large $\chi^{2}$ ) and the MSTR/MSTR-IE methods (higher MSE, very small $\chi^{2}$ ).

\section{References}

Barrada, J. R., Abad, F. J., \& Veldkamp, B. P. (2009). Comparison of methods for controlling maximum exposure rates in computerized adaptive testing. Psicothema, 21, 313-320.

Barrada, J. R., Abad, F. J., \& Olea, J. (2014). Optimal number of strata for the stratified methods in computerized adaptive testing. Spanish Journal of Psychology, 17, e48.

Camilli, G. (1994). Origin of the scaling constant in item response theory. Journal of Educational and Behavioral Statistics, 19, 293-295.

Chang, H., \& van der Linden, W. J. (2003). Optimal stratification of item pools in a-stratified computerized adaptive testing. Applied Psychological Measurement, 27, 262-274.

Chang, H., \& Ying, Z. (1999). A-stratified multistage computerized adaptive testing. Applied Psychological Measurement, 23, 211-222.

Chang, H., Qian, J., \& Ying, Z. (2001). $a$-Stratified multistage computerized adaptive testing with $b$ blocking. Applied Psychological Measurement, 25, 333-341. doi:10.1177/01466210122032181

Finkelman, M., Nering, L. M., \& Roussos, L. A. (2009). A conditional exposure control method for multidimensional adaptive testing. Journal of Educational Measurement, 46, 84-103.

Lee, Y. H., Ip, E. H., \& Fuh, C. D. (2008). A strategy for controlling item exposure in multidimensional computerized adaptive testing. Educational and Psychological Measurement, 68, 215-232.

Leung, C. K., Chang, H. H., \& Hau, K. T. (2002). Item selection in computerized adaptive testing: Improving the $a$-stratified design with the Sympson-Hetter algorithm. Applied Psychological Measurement, 26, 376-392.

Lord, F. M. (1980). Applications of item response to theory to practical testing problems. Hillsdale: Erlbaum.

Mulder, J., \& van der Linden, W. J. (2009). Multidimensional adaptive testing with optimal design criteria for item selection. Psychometrika, 74, 273-296.

Norweg, A., Ni, P., Garshick, E., O'Connor, G., Wilke, K., \& Jette, A. M. (2011). A multidimensional computer adaptive test approach to 
dyspnea assessment. Archives of Physical Medicine and Rehabilitation, 92, 1561-1569.

R Development Core Team. (2013). R: A language and environment for statistical computing. Vienna, Austria: R Foundation for Statistical Computing. ISBN 3-900051-07-0. Retrieved from www.R-project.org/

Segall, D. O. (1996). Multidimensional adaptive testing. Psychometrika, 61, 331-354.

Sympson, J. B., \& Hetter, R. D. (1985). Controlling item-exposure rates in computerized adaptive testing. In Proceedings of the 27th Annual Meeting of the Military Testing Association (pp. 973-977). San Diego: Navy Personnel Research and Development Center.

van der Linden, W. J., \& Veldkamp, B. P. (2004). Constraining item exposure in computerized adaptive testing with shadow tests. Journal of Educational and Behavioral Statistics, 29, 273-291.

van der Linden, W. J., \& Veldkamp, B. P. (2007). Conditional itemexposure control in adaptive testing using item-ineligibility probabilities. Journal of Educational and Behavioral Statistics, 32, 398-418.

Veldkamp, B. P., \& van der Linden, W. J. (2002). Multidimensional adaptive testing with constraints on test content. Psychometrika, 67, 575-588. doi:10.1007/BF02295132

Wang, C. (2015). On latent trait estimation in multidimensional compensatory item response models. Psychometrika, 80, 428-449. doi:10. 1007/S11336-013-9399-0
Wang, C., \& Chang, H. (2011). Item selection in multidimensional computerized adaptive testing - Gaining information from different angles. Psychometrika, 76, 363-384.

Wang, C., Chang, H., \& Boughton, K. A. (2011a). Kullback-Leibler information and its applications in multi-dimensional adaptive testing. Psychometrika, 76, 13-39.

Wang, C., Chang, H., \& Huebner, A. (2011b). Restrictive stochastic item selection methods in cognitive diagnostic computerized adaptive testing. Journal of Educational Measurement, $48,255-273$

Wang, C., Chang, H., \& Boughton, K. A. (2013). Deriving stopping rules for multidimensional computerized adaptive testing. Applied Psychological Measurement, 37, 99-122.

Yao, L. (2012). Multidimensional CAT item selection methods for domain scores and composite scores: Theory and applications. Psychometrika, 77, 495-523.

Yao, L., Pommerich, M., \& Segall, D. O. (2014). Using multidimensional CAT to administer a short, yet precise, screening test. Applied Psychological Measurement, 38, 614-631.

Zheng, Y., Chang, C. H., \& Chang, H. (2013). Content-balancing strategy in bifactor computerized adaptive patient-report outcome measurement. Quality of Life Research, 22, 491-499. 